\title{
Performance Evaluation of the TOF-Wall Detector of the FOOT Experiment
}

\author{
M. Morrocchi, N. Belcari, Member, IEEE, S. Bianucci, N. Camarlinghi, P. Carra, E. Ciarrocchi, M. De Simoni, \\ A. Del Guerra, Life Fellow, IEEE, M. Fischetti, M. Francesconi, L. Galli, A. C. Kraan, R. Mirabelli, A. Moggi, \\ S. Muraro, A. Profeti, M. Pullia, V. Rosso, A. Sarti, G. Sportelli, Senior Member, IEEE, G. Traini, R. Zarrella, \\ and M. G. Bisogni
}

\begin{abstract}
The correct quantification of the dose released in charged particle therapy treatments requires the knowledge of the double differential fragmentation cross section of particles composing both beam and target. The FOOT experiment aims at measuring these cross sections for ions of interest for charged particle therapy applications. The paper describes the performance of the TOF-Wall detector of the experiment. The detector is composed of two layers of $44 \mathrm{~cm} \times 2 \mathrm{~cm} \times 3 \mathrm{~mm}$ plastic scintillator bars (20 for each layer), arranged orthogonally and read out by silicon photomultipliers. The detector is designed to identify the charge of fragments ranging from protons to oxygen ions, with a maximum energy of $700 \mathrm{MeV} / \mathrm{u}$, by measuring the energy released in the scintillators and the time of flight with respect to a start counter. In this study, the detector was scanned with carbon ions of energy between $115 \mathrm{MeV} / \mathrm{u}$ and $400 \mathrm{MeV} / \mathrm{u}$ and with a $60 \mathrm{MeV}$ proton beam. The measurements show an energy resolution $\left(\sigma_{E} / \mu_{E}\right)$ between $6 \%$ and $4 \%$ and a contribution of the detector to the TOF system time resolution between 25 ps and 20 ps (standard deviation) for carbon ions and between 100 ps and 80 ps for protons.
\end{abstract}

Index Terms-Plastic scintillator, Silicon Photomultiplier, Time of Flight, Charged Particle Therapy, Nuclear Fragmentation.

\section{INTRODUCTION}

O $\mathrm{NE}$ of the main advantages of charged particle therapy is the release of a large fraction of energy in the region of the Bragg Peak, that allows treating deep seated tumors with a reduced dose to the surrounding healthy tissues. The estimation of the efficacy of the treatment relies on the radiobiological cell damage, which depends on the accuracy of the simulation of the dose absorbed by the tissue and on the correct estimation of the relative biological effectiveness

Manuscript received 27 July 2020; revised 7 November 2020. M. Morrocchi, N. Belcari, P. Carra, E. Ciarrocchi, A. Del Guerra, M. Francesconi, V. Rosso, G. Sportelli, R. Zarrella and M. G. Bisogni are with the Department of Physics, University of Pisa and INFN sezione di Pisa, Pisa, Italy. N. Camarlinghi is currently with LIST Spa, formerly was with the Department of Physics, University of Pisa and INFN sezione di Pisa, Pisa, Italy. A C. Kraan, L. Galli, A. Moggi, A. Profeti and S. Bianucci are with INFN sezione di Pisa, Pisa, Italy. M. Pullia is with Fondazione CNAO, Pavia, Italy. S. Muraro is with INFN sezione di Milano, Milano, Italy. G. Traini and R. Mirabelli are with Department of Physics, Sapienza University of Rome and INFN Sezione di Roma, and also with Museo Storico della Fisica e Centro Studi e Ricerche E. Fermi, Roma, Italy. M. De Simoni is with Dipartimento di Fisica, Sapienza University of Rome and INFN Sezione di Roma, Roma, Italy. M. Fischetti is with Dipartimento di Scienze di Base e Applicate per l'Ingegneria, Sapienza University of Rome and INFN Sezione di Roma, Roma, Italy. A. Sarti is with Dipartimento di Scienze di Base e Applicate per l'Ingegneria, Sapienza University of Roma, INFN Sezione di Roma and Museo Storico della Fisica e Centro Studi e Ricerche E. Fermi, Roma, Italy.(e-mail: matteo.morrocchi@unipi.it).
(RBE) of the particle [1]. In particular, a crucial role in the RBE calculation is played by the fragmentation of the nuclei composing the tissues along the beam path and, for therapeutic ion beams with $Z>1$, also of the beam itself. In proton therapy, an RBE of 1.1 is generally assumed in the model of the treatment planning system (TPS) [2]. However, this value underestimates the damage induced by inelastic interactions of the beam with the target nuclei before the Bragg peak, which generate fragments with high linear energy transfer (LET) and short range, resulting in a high local dose and in a broadening of the dose distribution [3]. Since these inelastic interactions occur almost uniformly in the whole proton range [4], fragments with a high LET are also produced in the healthy tissue before the Bragg peak. In the case of ion therapy, nuclear fragmentation of the primary beam produces secondary ions with lower atomic number and longer range, resulting in a tail in the dose profile beyond the Bragg peak [5]. To properly evaluate the healthy tissue damage, fragmentation needs to be accurately included in the TPS by means of a full set of interaction cross-sections. Even though some experiments were carried out to measure the fragmentation probability of ions like carbon or helium [6]-[9] and fragmentation probabilities were tabulated for some ions and various energies and angles [10]-[13], a full database of differential and double differential cross sections, covering the whole spectrum of therapeutic energies, is still missing.

The FOOT (FragmentatiOn Of Target) experiment aims at measuring the double differential fragmentation cross-section of nuclei relevant in charged particle therapy. The experimental apparatus will be composed of a set of detectors that track the fragments produced in a target to identify their mass, charge, energy and emission angle [14]. Beam fragmentation is studied with beams of helium, carbon, or oxygen ions with energies in the range $200 \mathrm{MeV} / \mathrm{u}-400 \mathrm{MeV} / \mathrm{u}$ delivered at low intensities (few $\mathrm{kHz}$ ), so to study every single particle. For target fragmentation in proton therapy, due to the short range of the fragment in the patient reference frame, an inverse kinematic approach is used, thus accelerating carbon and oxygen beams onto an hydrogen-enriched target. The FOOT apparatus will also be used with higher energy beams (up to $700 \mathrm{MeV} / \mathrm{u}$ ) for radioprotection studies in long space missions. A scheme of the experimental set-up is represented in Fig. 1.

Primary particles are detected by a start counter [15] that registers the start time, then they are tracked by a beam monitor (a drift chamber composed of 12 layers with alternated 

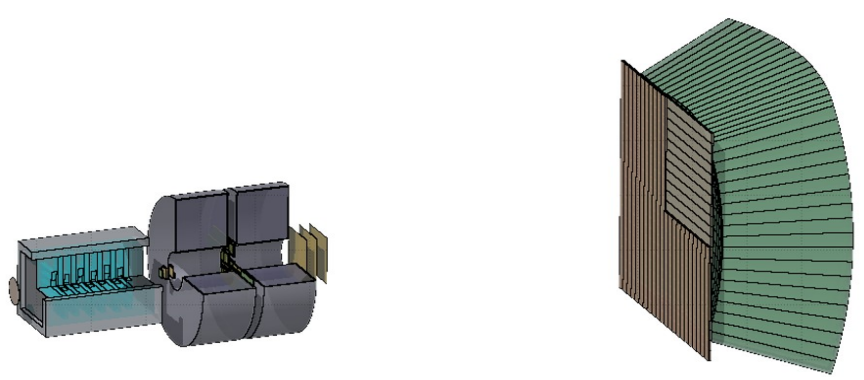

Fig. 1. Scheme of the FOOT experimental apparatus.

horizontal and vertical wires) before interacting with a thin target composed of carbon or $\mathrm{C}_{2} \mathrm{H}_{4}$. A magnetic spectrometer, composed of two magnets and layers of pixel detectors and silicon strips, is used to determine the momentum of the primary particle and of all fragments. A detector named TOFWall (TW) and composed of plastic scintillators measures the deposited energy and the Time of Flight (TOF) with respect to the start counter. A BGO calorimeter measures the kinetic energy.

The TOF-Wall detector, in combination with the startcounter, provides the charge of each fragment and the angular deflection with respect to the beam axis. This information is used in the global event reconstruction to retrieve the particle mass and to obtain the double-differential crosssection for each fragmentation process. The TOF-Wall detector is composed of two layers of plastic scintillator bars orthogonally arranged and read-out at both ends by silicon photomultipliers (SiPMs). The active area of the detector is 40 $\mathrm{cm} \times 40 \mathrm{~cm}$, ensuring an angular coverage of approximately $10^{\circ}$. To achieve the requirements of the FOOT experiment in terms of $Z$ resolution, the TOF-Wall detector needs to reach an energy resolution (expressed as the ratio between the standard deviation over the mean of the reconstructed energy distribution, $\sigma_{E} / \mu_{E}$ ) of the order of $5 \%$, and a time resolution for the TOF system below $100 \mathrm{ps}$ (standard deviation). The constraint on the detector time resolution is partially dictated by the maximum acceptable distance between start-counter and TOF-Wall, required to allow the FOOT apparatus to fit the experimental rooms of different ion therapy facilities. However, a maximum distance of approximately 1 meter is required to fully cover the fragment angular aperture. For heavy fragments, since the angular aperture is limited, a larger distance of the TW detector can be used, thus improving the system time resolution. The thickness of the scintillator layers has been minimized to reduce the secondary fragmentation probability in the detector to a few percents. Given the different types of fragments interacting in the detector (from ${ }^{1} \mathrm{H}$ to ${ }^{16} \mathrm{O}$ ), a wide dynamic range was also required.

Plastic scintillators are widely employed in TOF measurements and $Z$-identification thanks to their fast scintillation time, relatively high light yield, low cost, and capability to instrument large volumes. As an example, a TOF-Wall detector with an active area of $1.2 \times 1.2 \mathrm{~m}^{2}$ has been developed with 30 scintillator bars $\left(120 \times 4 \times 1 \mathrm{~cm}^{3}\right)$ read-out at both ends by photomultiplier tubes [16]. A time resolution of about
$120 \mathrm{ps}$ has been achieved irradiating the detector with a 310 $\mathrm{MeV} / \mathrm{u}{ }^{40} \mathrm{Ar}$ beam. Plastic scintillator bars are employed also in space experiments [17], [18] for the charge identification of cosmic nuclei. The main drawback of plastic scintillators in $Z$ identification experiments is their non-linear response due to the saturation of the scintillating centers [19], which requires an accurate calibration to quantify the energy released in the detector.

We have already reported the performance evaluation of small-scale prototypes of the TOF-Wall detector [20], [21] which allowed us to optimize its architecture and to demonstrate that fragments produced by carbon ions impinging onto a plastic target can be correctly identified [22], [23]. In this paper, we describe the design of a full scale TW prototype and report its performance scanning the detector with proton and carbon beams in terms of energy and time resolution; we also studied the signal attenuation along the bar. Finally, we present a method to reconstruct the interaction position in the detector and the spatial resolution obtained.

\section{Materials AND MethodS}

\section{A. Detector Structure}

The TOF-Wall detector is composed of two layers of plastic scintillator bars (EJ-200 by Eljen Technology, Sweetwater, Texas), arranged orthogonally and wrapped with ESR specular reflector (Fig. 2). Each bar is $3 \mathrm{~mm}$ thick, $20 \mathrm{~mm}$ wide, and $440 \mathrm{~mm}$ long. Each layer is composed of 20 bars, giving a global sensitive area of $40 \mathrm{~cm} \times 40 \mathrm{~cm}$. Each bar end is coupled to four silicon photomultipliers (MPPC by Hamamatsu) with $3 \mathrm{~mm} \times 3 \mathrm{~mm}$ active area and $25 \mu \mathrm{m}$ microcell pitch. The four SiPMs are connected as the parallel of two branches, each one composed of the series of two devices (Fig. 3). Each side of the bar is read-out as a single channel (corresponding to a dynamic range of 57600 cells). This configuration reduces the total capacitance and, consequently, the rise-time of the signal at the expense of a factor- 2 loss in the collected charge. SiPMs belonging to the same channel are grouped according to similar breakdown voltage and dark-noise values. Each group of four SiPMs is biased independently at $5 \mathrm{~V}$ overvoltage for each SiPM. The 80 signals are digitized at 4 Gsamples/s with 12-bit resolution by the WaveDAQ electronics system [24], hosting the Domino Ring Sampler DRS ASIC [25], [26]. The WaveDAQ system hosts an amplification stage that allows to tune the amplitude in a range of $100 \times-0.5 \times$. A total of 1024 samples are collected for each waveform. The detector is mounted on a set of motorized linear stages to scan the whole sensitive area with a fixed beam.

\section{B. Measurements Set-Up}

In this study, the detector was irradiated at the Centro Nazionale di Adroterapia Oncologica (CNAO - Pavia, Italy) with carbon beams of three energies $(115 \mathrm{MeV} / \mathrm{u}, 260 \mathrm{MeV} / \mathrm{u}$ and $400 \mathrm{MeV} / \mathrm{u}$ ) and with a $60 \mathrm{MeV}$ proton beam. The detector was moved as shown in Fig. 4. In this study, the bars in the front layer were arranged horizontally while those of the rear layer were vertical. Considering bar IDs going from \#0 to \#19 in the rear layer and from \#20 to \#39 in the front layer, 


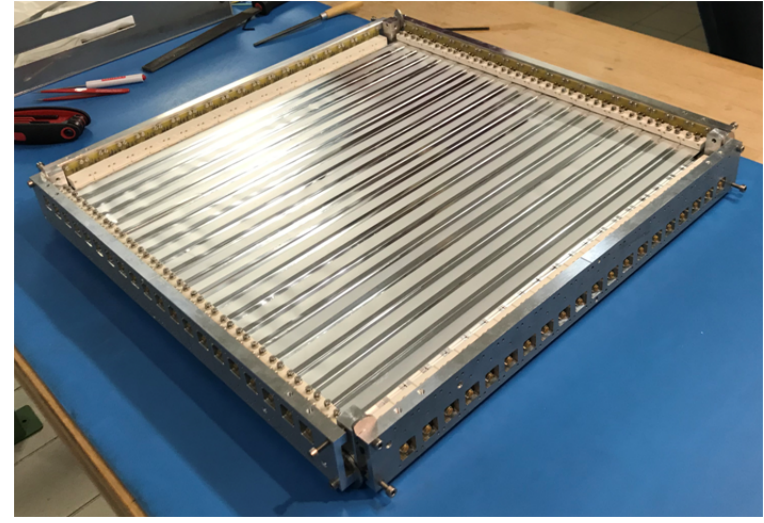

Fig. 2. Picture of the TOF-Wall detector.
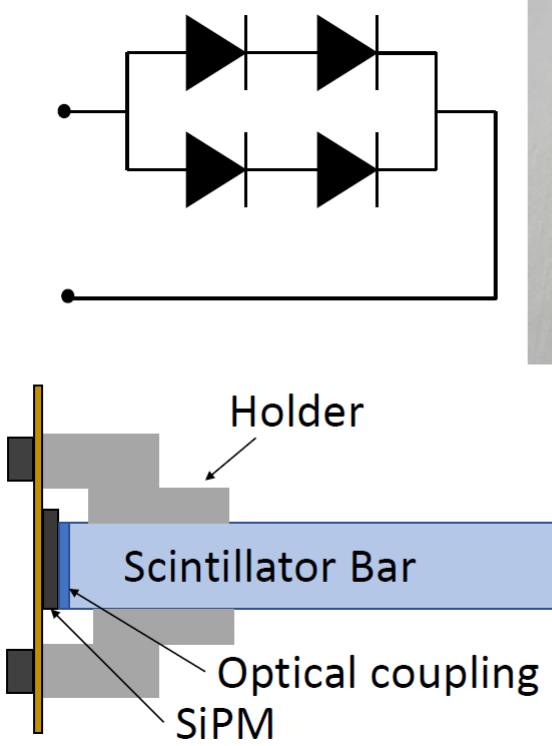

Fig. 3. Left, scheme of the read-out circuitry. Right, Picture of a SiPM board. Bottom, scheme of the coupling between SiPMs and scintillator bars.

bar \#9 of the rear layer was irradiated in 18 different positions so that bars from \#21 to \#38 of the front layer were irradiated in their centers. A similar scan was performed on bar \#30 of the front layer, irradiated at the intersections between bars \#1 to \#18 of the rear layer. In the following, bars \#9 and \#30 will be referred to as reference bars of the two layers. The beam delivery rate was of the order of $10^{4}$ particles per second to avoid event pile-up. The event acquisition rate during the session was about $10^{3}$ events/s and a total of $5 \cdot 10^{3}$ events for each point were acquired. The front-end gain was set to 1.0 for all beam energies. Events were saved every time at least one channel for each layer was triggered.

\section{Data Processing and Analysis}

For each triggered channel the whole waveform was recorded. An example of a waveform is shown in Fig. 5. The rise time of the signal is reported in Table II for each beam. The area of the signal was used to evaluate the collected charge, while the constant fraction discriminator method with

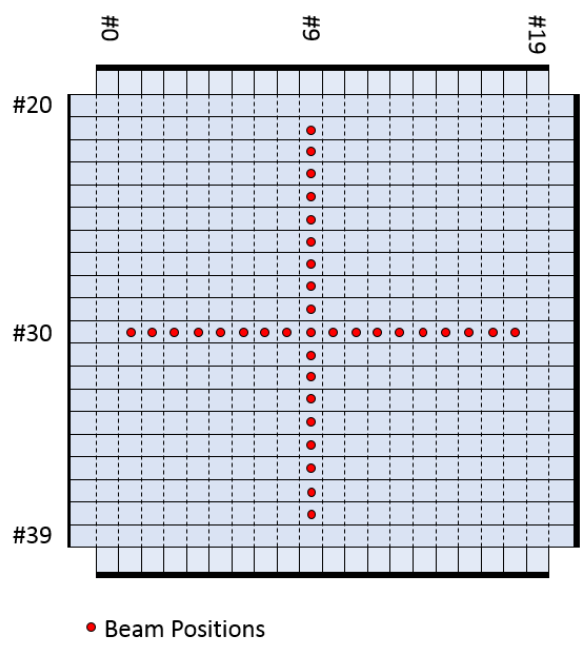

Fig. 4. Scheme of the irradiation performed during the test of the detector. Dots correspond to each nominal position of the beam during the scan.

threshold at $30 \%$ of the peak was applied to define the timestamp. To align each triggered channel, the clock sent to the DRS4 ASICs was digitized and read-out. The skew between the clocks that were sent to the ASICs for signal digitization was evaluated and corrected in the analysis. Mismatch in cabling was not taken into account in the analysis and will require a custom calibration with the start counter detector. The total collected charge of each bar, $Q_{i}$, was determined as the square root of the product of the charge collected at the two ends $Q_{i, 1}$ and $Q_{i, 2}$ where the numbers 1 and 2 indicate the two ends of each bar, and $i$ is the bar ID:

$$
Q_{i}=\sqrt{Q_{i, 1} \cdot Q_{i, 2}}
$$

$Q$ was evaluated according to eq. 1 to partially correct for the light attenuation along the bar. The timestamp of the $i-t h$ bar, $T_{i}$, was evaluated as the mean of the timestamps of the signals generated at the two ends, $T_{i, 1}$ and $T_{i, 2}$ :

$$
T_{i}=\frac{T_{i, 1}+T_{i, 2}}{2}
$$

To convert the collected signal to the energy released by the particle in the bar, a calibration procedure was implemented, using a model based on Birks' law:

$$
Q_{i}=\frac{s_{i} \cdot E_{i}}{1+k_{i} \cdot E_{i}}
$$

where $s$ is the signal generated per unit of released energy when there are no saturation effects, $k$ is the coefficient that accounts for the scintillator saturation and $E_{i}$ are the simulated energies released in the bar (Table I). The effects related to the SiPM saturation, which follow the model described in [27], have not been included to avoid too many free fit parameters, this effect is then included in the value of the $k$ parameter. A more accurate model could be applied by measuring more energies for a larger number of ions. Even though, in principle, the value of $k$ should be the same for all the bars, it has been found independently for each one and the uniformity of the obtained values has been then investigated. The energy $E_{i}$ 
TABLE I

MONTE CARLO VALUES OF THE ENERGY RELEASED IN THE FRONT AND REAR SCINTILLATOR BARS BY THE VARIOUS BEAMS.

\begin{tabular}{|l|c|c|}
\hline & \multicolumn{2}{|c|}{ Released Energy (MeV) } \\
Beam & Front Layer & Rear Layer \\
\hline $\mathbf{p ~ ( 6 0 )}$ & 3.4 & 3.6 \\
C (115) & 78.5 & 82.5 \\
C (260) & 42.6 & 42.9 \\
C (400) & 33.4 & 33.5 \\
\hline
\end{tabular}

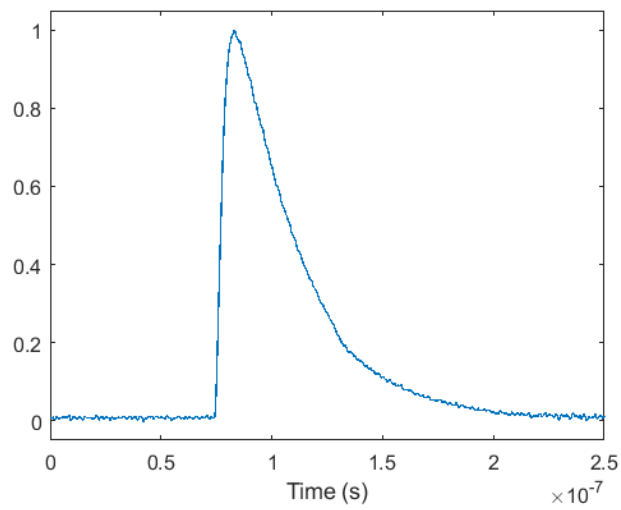

Fig. 5. Example of a waveform, with normalized amplitude, obtained with $260 \mathrm{MeV} / \mathrm{u}$ carbon beam at the center of the detector.

released in the bar was estimated for each beam by means of Monte Carlo simulations implemented in FLUKA [28]. In the simulations, the detectors used for the beam monitoring before the TOF-Wall detector was also taken into account for the correct estimation of the released energy. The values are reported in Table I.

The following quantities were studied:

1) Attenuation length: The attenuation length was evaluated for the two reference bars of the two layers by using the signals collected at both ends as a function of the beam distance from the SiPMs. The collected light as a function of the distance from the scintillation position was modeled according to the equation:

$$
Q=a \cdot e^{-\frac{x}{l}}+c
$$

where $Q$ is the signal at the end of the bar, $l$ is the attenuation length due to the interaction with the wrapping and to the self-absorption of the scintillator, and $c$ represents a constant contribution independent of the interaction point. The uniformity of the collected signal as a function of the beam position was also evaluated.

2) Energy Resolution: For each irradiated point, the collected signal was calibrated according to eq. 3 . The signal was then converted to released energy, and the energy resolution

TABLE II

RISE TIME (10\%-90\%) OF THE SIGNAL FOR EACH PARTICLE BEAM

\begin{tabular}{|c|c|c|c|c|}
\hline & $\begin{array}{c}p 60 \\
(\mathrm{MeV})\end{array}$ & $\begin{array}{c}C 400 \\
(\mathrm{MeV} / \mathrm{u})\end{array}$ & $\begin{array}{c}C 260 \\
(\mathrm{MeV} / \mathrm{u})\end{array}$ & $\begin{array}{c}C 115 \\
(\mathrm{MeV} / \mathrm{u})\end{array}$ \\
\hline Rise Time (ns) & $5.3 \pm 0.5$ & $4.8 \pm 0.1$ & $4.8 \pm 0.1$ & $4.7 \pm 0.1$ \\
\hline
\end{tabular}

TABLE III

RATIO BETWEEN THE EXPONENTIAL AND THE CONSTANT COMPONENTS, FOR THE FOUR BEAMS AND FOR THE TWO ENDS OF THE TWO REFERENCE BARS OF THE TWO LAYERS.

\begin{tabular}{|l|c|c|c|c|}
\cline { 2 - 5 } \multicolumn{1}{c|}{} & \multicolumn{4}{c|}{$\boldsymbol{a / c}$} \\
\cline { 2 - 5 } \multicolumn{1}{c|}{} & $\begin{array}{c}\boldsymbol{C ~ 4 0 0} \\
(\mathbf{M e V} / \boldsymbol{u})\end{array}$ & $\begin{array}{c}\boldsymbol{C ~ 2 6 0} \\
(\mathbf{M e V} / \boldsymbol{u})\end{array}$ & $\begin{array}{c}\boldsymbol{C ~ 1 1 5} \\
(\mathbf{M e V} / \boldsymbol{u})\end{array}$ & $\begin{array}{c}\boldsymbol{p ~ 6 0} \\
(\mathbf{M e V} / \boldsymbol{u})\end{array}$ \\
\hline Left Front & $2.9 \pm 0.2$ & $2.8 \pm 0.2$ & $2.8 \pm 0.2$ & $2.8 \pm 0.2$ \\
Right Front & $2.6 \pm 0.2$ & $2.7 \pm 0.2$ & $2.6 \pm 0.2$ & $2.5 \pm 0.2$ \\
Top Rear & $2.2 \pm 0.2$ & $2.3 \pm 0.2$ & $2.1 \pm 0.2$ & $2.3 \pm 0.2$ \\
Bottom Rear & $2.5 \pm 0.2$ & $2.5 \pm 0.2$ & $2.4 \pm 0.2$ & $2.3 \pm 0.2$ \\
\hline
\end{tabular}

$\sigma_{E} / \mu_{E}$ was evaluated for each layer individually and for the whole TOF-Wall by combining the information of both layers.

3) Time resolution: The time resolution between the two layers $\left(\sigma_{i, j}\right)$ was evaluated considering the distribution of the difference between the timestamps of the front and the rear layer:

$$
\Delta T_{i, j}=T_{i}-T_{j}
$$

where $T_{j}$ is the timestamp of the triggered bar in the rear layer (with $0 \leq j \leq 19$ ) and $T_{i}$ is the timestamp of the bar in the front layer (with $20 \leq i \leq 39$ ). The TOF-Wall timestamp $\left(T_{T W}\right)$ was defined as:

$$
T_{T W}=\left(T_{i}+T_{j}\right) / 2
$$

and provides the arrival time of the fragment in the TW. The resolution of the TOF-Wall detector $\sigma_{T W}$, assuming a similar resolution of both layers in the fragment timestamp, is half that obtained between the two bars $\left(\sigma_{T W}=\sigma_{i, j} / 2\right)$. In fact, $\sigma\left(T_{i}-T_{j}\right)=\sigma\left(T_{i}+T_{j}\right)$, therefore $\sigma\left(T_{T W}\right)=\sigma\left(T_{i}+T_{j}\right) / 2=$ $\sigma\left(T_{i}-T_{j}\right) / 2=\sigma_{i, j} / 2$.

4) Interaction position: If only one fragment interacts in the detector, the interaction position can be easily reconstructed by identifying the intersection between the front and the rear bar that are triggered by the ion. However, if two or more fragments interact simultaneously, the identification of the interacting position of each ion is not trivial. Anyway in the final FOOT set-up the positions can be identified by merging the information from the tracking system or from the pixelated calorimeter positioned right after the TW. We investigated here the capability to disentangle multiple events by measuring the difference in timestamps of the signal collected at the two ends of each bar $\left(\Delta T_{i}=T_{i, 1}-T_{i, 2}\right)$.

\section{RESULTS}

\section{A. Attenuation length}

The signal at one end of the reference bar of the front layer is shown in Fig. 6 as a function of the distance between the beam interaction point and the SiPM board. Table III summarizes the results obtained in terms of the ratio $a / c$ and Table IV reports the values obtained for the attenuation lengths $l$.

The ratio between the two contributions of eq. 4 is constant for each bar. The optical attenuation length is constant for each channel, with the exception of the proton case, which gives a systematically smaller value. The right side of the front reference bars gives values of the attenuation length that 
TABLE IV

ATTENUATION LENGTH OBTAINED FOR THE FOUR BEAMS AND FOR THE TWO ENDS OF THE TWO REFERENCE BARS OF THE TWO LAYERS.

\begin{tabular}{|l|c|c|c|c|}
\cline { 2 - 5 } \multicolumn{1}{c|}{} & \multicolumn{4}{c|}{$\boldsymbol{l}(\boldsymbol{c m})$} \\
\cline { 2 - 5 } & $\begin{array}{c}\boldsymbol{C ~ 4 0 0} \\
(\mathbf{M e V / u})\end{array}$ & $\begin{array}{c}\boldsymbol{C ~ 2 6 0} \\
(\mathbf{M e V / u})\end{array}$ & $\begin{array}{c}\boldsymbol{C ~ 1 1 5} \\
(\mathbf{M e V / u})\end{array}$ & $\begin{array}{c}\boldsymbol{p ~ 6 0} \\
(\mathbf{M e V / u})\end{array}$ \\
\hline Left Front & $17.9 \pm 0.7$ & $18.0 \pm 0.6$ & $19.2 \pm 0.7$ & $15.8 \pm 0.6$ \\
Right Front & $22.6 \pm 0.8$ & $23.2 \pm 0.7$ & $23.6 \pm 0.8$ & $20.0 \pm 0.8$ \\
Top Rear & $16.5 \pm 0.6$ & $17.9 \pm 0.7$ & $17.1 \pm 0.6$ & $14.2 \pm 0.6$ \\
Bottom Rear & $17.0 \pm 0.6$ & $18.2 \pm 0.7$ & $17.7 \pm 0.7$ & $14.7 \pm 0.6$ \\
\hline
\end{tabular}

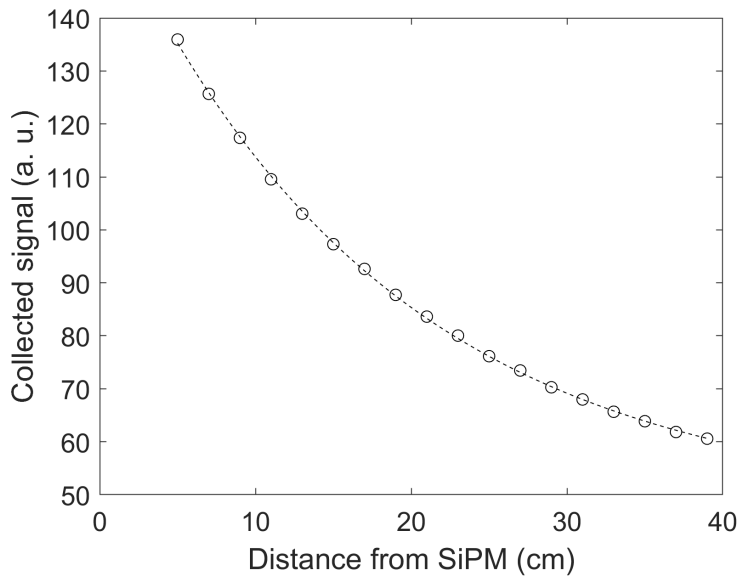

Fig. 6. Signal at one end of the reference bar of the front layer as a function of the distance between the beam and the photo-detector obtained with 260 $\mathrm{MeV} / \mathrm{u}$ carbon ions.

are consistent among each other, but not in agreement with the rest of the measurements. This behaviour is still under investigation.

Figure 7 shows the total signal calculated with eq. 1 of the reference bar of the front layer for the four beams. Since the signal at each end of the bar is not modeled by a single exponential attenuation, the value of the total signal is not constant along the bar. A difference of about $5 \%(15 \%)$ is observed between the signals when the particle interacts at the center of the bar and when the particle interacts near the left (right) end of the bar.

\section{B. Energy Resolution}

An example of the mean signal as a function of the energy released in the bar is shown in Fig. 8 for one representative beam position (center bar \#9). The signal was determined using eq. 1 and fitted using eq. 3 . The fit parameters $k$ (blue) and $s$ (red) are reported in Fig. 9 for the two reference bars of the front and rear layer. For each bar, results are shown for each of the 18 beam interaction positions reported in Fig. 4. As can be seen, the saturation parameters are constant within a few percent along the bar except for the first and last two measurements. Since in these cases the beam was closer to one of the two ends, we can expect a higher contribution of the SiPM saturation. The $s$ parameter reproduces the trend of the total signal as a function of the irradiation position already shown in Fig. 7. Figure 10 represents the overall distribution of the $k$ parameter in all bars for all the irradiated positions.

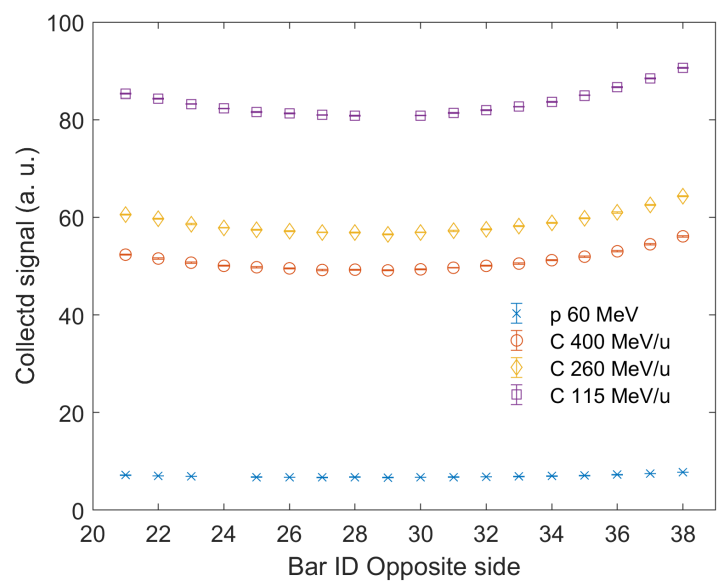

Fig. 7. Total signal in the reference bar of the rear layer as a function of the beam position (expressed as the ID of the triggered bar in the opposite layer) for the four beams.

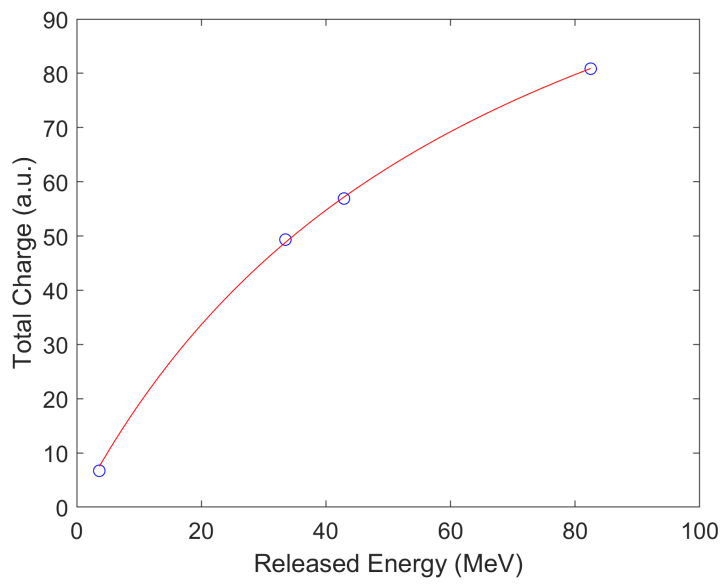

Fig. 8. Signal as a function of the energy released in the center bar \#9. The fit performed according to the Birks' equation is also reported.

As can be seen from this figure, the value is uniform in the whole detector, with the exception of few outliers. The energy resolution has been then evaluated for each bar and for each beam energy. Figure 11 shows the results obtained in terms of energy resolution for front and rear layer in all the irradiated positions. The result obtained by merging together the energy collected on the two layers is also reported. An energy resolution below 7\% has been obtained for each individual layer with carbon ions, and a resolution below 5\% is achieved when the energies collected in both layers were averaged.

\section{Time resolution}

Figure 12 shows the time resolution obtained between the reference bar of the front layer and each bar of the rear layer irradiated in coincidence during the scan. The time resolution between the two layers, $\sigma_{i, j}$, achieved with carbon ions is between $35 \mathrm{ps}$ and $50 \mathrm{ps}$, slightly degrading at the ends of the bars. This corresponds to a contribution of the TOF-Wall detector between 20 ps and 25 ps to the time resolution of 


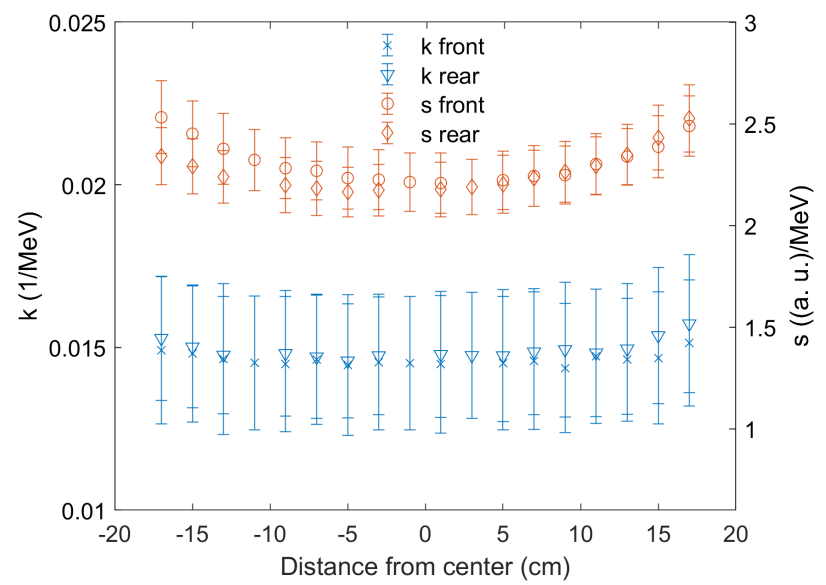

Fig. 9. Fit parameters obtained for the reference bars of the front layer.

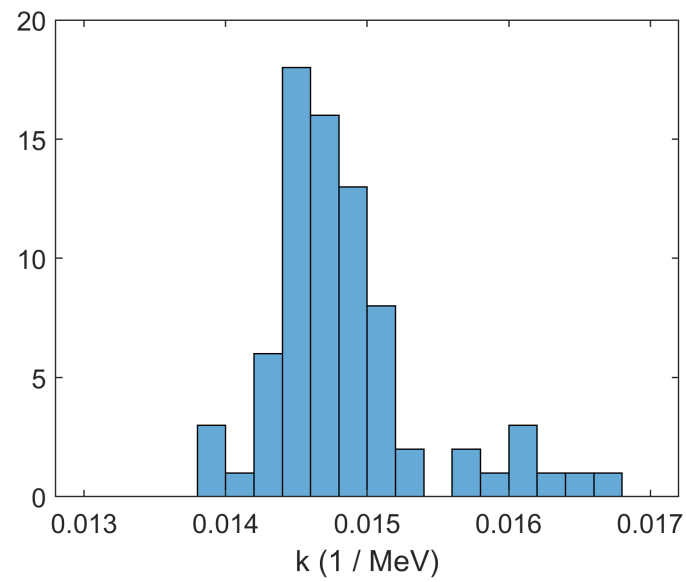

Fig. 10. k-parameter measured in all the investigated positions of the two layers.

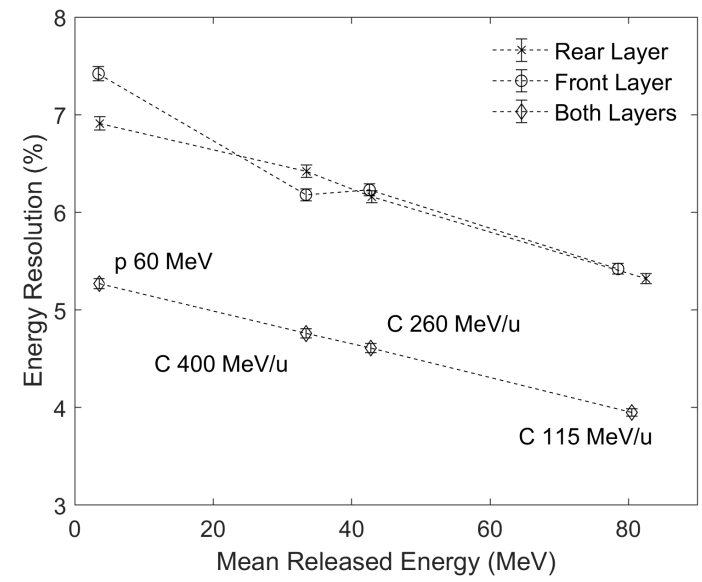

Fig. 11. Energy resolution obtained independently for each layer, and averaging the energy reconstructed on the two layers. The resolution is expressed as the ratio between the standard deviation and the center of the distribution.

the TOF apparatus. The best performance was achieved, as expected, with $115 \mathrm{MeV} / \mathrm{u}$ ions, when a higher energy is

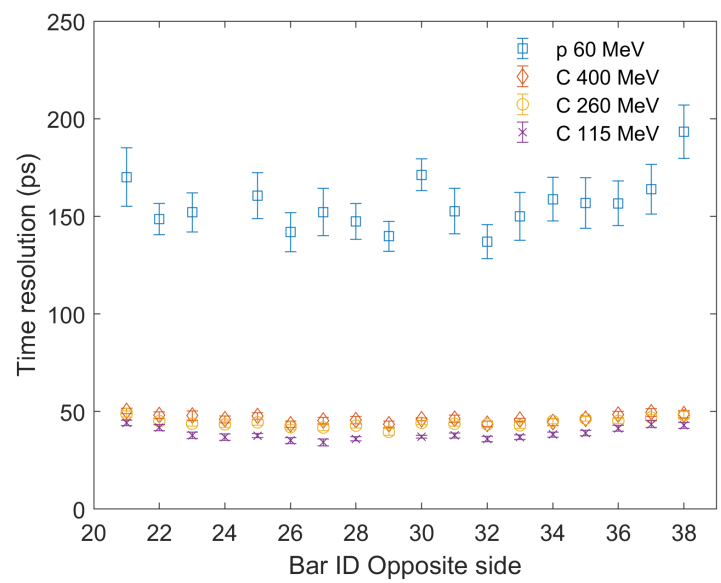

Fig. 12. Time resolution obtained between the reference bar of the rear layer (bar \#9) and each bar of the front layer. The resolution is expressed as the standard deviation of the difference in the reconstructed hit time distribution of the two layers. The acquisition with $115 \mathrm{MeV} / \mathrm{u}$ carbon ions at the position corresponding to bar \#29 and with $60 \mathrm{MeV}$ protons with bar \#24 are missing due to problems occurred in those runs.

released. An average time resolution of $160 \mathrm{ps}$ was achieved with proton beams between the two layers.

\section{Interaction position identification}

Figure 13 shows the timestamp difference distribution between the two sides of the reference bar of the front layer, for five representative beam positions of the $260 \mathrm{MeV} / \mathrm{u}$ carbon beam (solid line) and of the $60 \mathrm{MeV}$ proton beam (dotted line). The distance of the beam from the center of the detector is indicated in the text box above each peak. Figure 14 reports the center of all the distributions for the 4 different beams as a function of the position of the beam along the reference bar. $\Delta T$ depends linearly on the interaction position in the bar, and returns a propagation velocity of the light in the bars of $v_{l}=14.83 \pm 0.02 \mathrm{~cm} / \mathrm{ns}$. Between the two ends of the bar, we obtained average time resolutions $\sigma_{\Delta T}=72 \pm 10 \mathrm{ps}$ and $\sigma_{\Delta T}=260 \pm 20 \mathrm{ps}$, for $260 \mathrm{MeV} / \mathrm{u}$ carbon ions and $60 \mathrm{MeV}$ protons, respectively. These results can be converted in terms of spatial resolution $\left(F W H M_{x}\right)$ along the bar as:

$$
F W H M_{x}=2.35 \cdot \frac{v_{l} \cdot \sigma_{\Delta T}}{2}
$$

To estimate the detector spatial resolution along the bar, the contribution of the beam width [29] has been subtracted in quadrature. Since the beam dimensions were not measured directly, the actual values during the data taking could be slightly different. The results are reported in Table V.

\section{DISCUSSION}

In this study, we reported the energy and time performance of the final TOF-Wall detector of the FOOT experiment. In particular, we demonstrated that a resolution in the energy deposited in the ToF-Wall detector close to $4 \%$ can be achieved with carbon ions with $115 \mathrm{MeV} / \mathrm{u}$ kinetic energy impinging onto the detector. To reach this performance, an independent calibration for each pair of bars in the two layers needs to 
TABLE V

FWHM OF THE SPATIAL DISTRIBUTION OF THE RECONSTRUCTED INTERACTION POSITION ALONG THE BAR, THE BEAM WIDTH AND THE RESULTS CORRECTED FOR THIS PARAMETER ARE ALSO REPORTED

\begin{tabular}{|c|c|c|c|}
\hline Beam & $\begin{array}{c}\text { raw FWHM } \\
(\mathbf{m m})\end{array}$ & $\begin{array}{c}\text { Beam FWHM } \\
(\mathrm{mm})\end{array}$ & $\begin{array}{l}\text { Sp. Res. } \\
\text { (mm) }\end{array}$ \\
\hline p $60 \mathrm{MeV}$ & $45.3 \pm 4.1$ & 23.4 & $38.8 \pm 5.0$ \\
\hline C $400 \mathrm{MeV} / \mathrm{u}$ & $13.4 \pm 0.9$ & 4.5 & $12.6 \pm 0.9$ \\
\hline C $260 \mathrm{MeV} / \mathrm{u}$ & $12.5 \pm 1.1$ & 5.2 & $11.4 \pm 1.1$ \\
\hline C $115 \mathrm{MeV} / \mathrm{u}$ & $13.2 \pm 1.7$ & 9.5 & $9.2 \pm 2.3$ \\
\hline
\end{tabular}

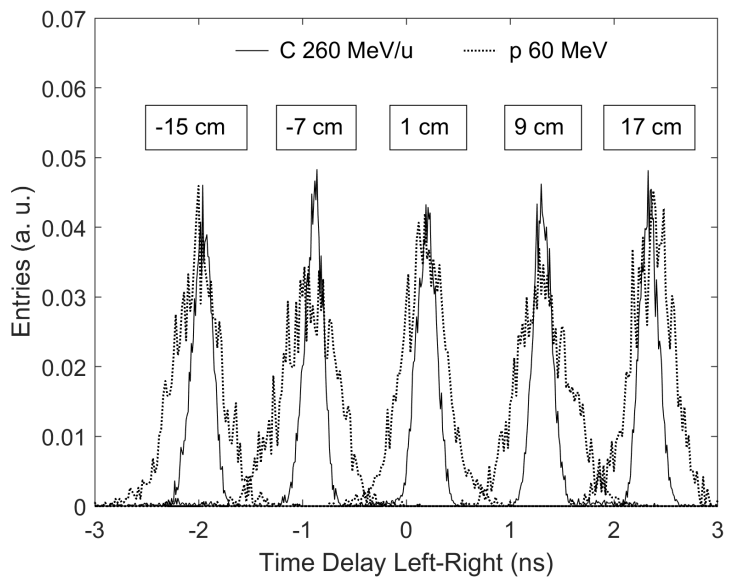

Fig. 13. Distribution of the timestamps difference between the left and right side of the reference rear bar (bar \#9) for each beam position. The area of each distribution has been normalized. The distributions refer to the scans with $260 \mathrm{MeV} / \mathrm{u}$ carbon ions and $60 \mathrm{MeV}$ protons.

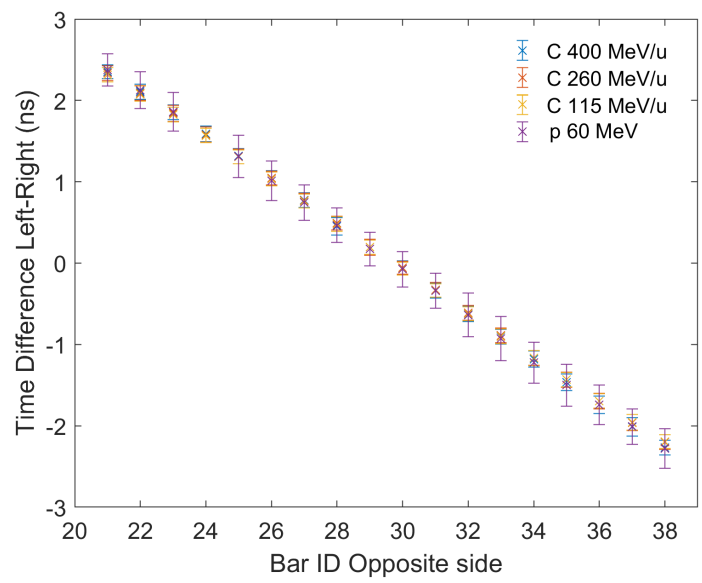

Fig. 14. Mean delay between the two ends of the reference rear bar for each beam position and for each energy and particle.

be performed with different beam energies and with different particles. If all the 400 possible bar pairs are investigated, considering $5 \cdot 10^{3}$ events for each position, as used in this experiment, and an acquisition rate of $2 \cdot 10^{3} \mathrm{~Hz}$, a total acquisition time of approximately 40 minutes is required to scan the whole detector with one beam configuration (assuming 3 seconds dead time between each position), giving a total time of less than three hours for the four beam configurations of this study. For an exhaustive calibration of the detector, all the detectable ions would be needed, due to the dependence of the saturation model of the scintillation on the charge of the impinging ion [30]. The need for a point-by-point calibration is further suggested by the dependence of the amount of collected light at the two ends of the bar on the fragment interaction position.

A time resolution below $50 \mathrm{ps}$ (standard deviation) between the two layers was achieved with carbon ions. Considering that the timestamp of the TOF-Wall detector is given by the average timestamp of the two layers, an improvement in time resolution of a factor 2 is expected, suggesting a time resolution between $20 \mathrm{ps}$ and $25 \mathrm{ps}$ for the detector with carbon ions. For protons, we expect a time resolution between $80 \mathrm{ps}$ and $100 \mathrm{ps}$.

These results suggest the possibility to investigate a configuration of the detector in which the two layers have different settings, i. e., one optimized for high energy deposits, with the current settings, and the other optimized for proton interactions, with higher front-end gain. This asymmetric configuration will be investigated in future tests since it would allow improving the time resolution for protons, at the expenses of a slight reduction in energy resolution, when the information of the two layers will not be merged together.

It was demonstrated that the interaction position can be identified even with the information of a single layer, as long as each bar will not be hit by more than one fragment. This property allows discriminating multiple interactions that occur in the detector without the correlation of the TOF-Wall detector information with the data of other detectors, as long as each event involves a different couple of horizontal-vertical bars.

\section{CONClusion}

The TOF-Wall detector for the FOOT experiment has been built and its performance in terms of energy resolution, time resolution, and hit position identification has been studied using proton and carbon ion beams. The time performance is compliant with the requirements of the FOOT experiment. In particular, a contribution of the TOF-Wall to the TOF resolution between $20 \mathrm{ps}$ and $25 \mathrm{ps}$ (standard deviation) is expected for carbon ions. The energy resolution $(\sigma / \mu)$ is within the requirements of $5 \%$ if the charge information of both layers is used. It has also been shown that multiple interactions can be discriminated using the information of the TOF-Wall detector alone. Future studies will be dedicated to investigating the detector charge identification capability.

\section{REFERENCES}

[1] Relative Biological Effectiveness (RBE), Quality Factor (Q), and Radiation Weighting Factor (wR). HICRP, ICRP Publication 92. Ann. ICRP 33 (4), 2003.

[2] H. Paganetti, et al., Relative biological effectiveness (RBE) values for proton beam therapy. Variations as a function of biological endpoint, dose, and linear energy transfer Phys. Med. Biol., vol. 59, issue 22, pp. 419-472, November 2014.

[3] N. Matsufuji, et al., Spatial fragment distribution from a therapeutic pencil-like carbon beam in water, Phys. Med. Biol., vol. 50, issue 14, pp.3393-3403, July 2005.

[4] R. Pleskac, et al., "The FIRST experiment at GSI", Nuclear Inst. and Methods in Physics Research, A, vol. 678, pp. 130-138, June 2012. 
[5] L. Sihver, D. Schardt and T. Kanai, Depth-dose distributions of highenergy carbon, oxygen and neon beams in water, Japan J. Med. Phys., vol. 18, issue 1, pp. 1--21, January 1998.

[6] M. Toppi, et al., Measurement of fragmentation cross sections of $12 \mathrm{C}$ ions on a thin gold target with the FIRST apparatus, Phys. Rev. C, vol. 93, June 2016, Art. No. 064601.

[7] M. Marafini, et al., Secondary radiation measurements for particle therapy applications: Nuclear fragmentation produced by $4 \mathrm{He}$ ion beams in a PMMA target. Phys. Med. Biol., vol. 62, issue 4, pp. 1291-1309, February 2017.

[8] J. Dodouet, et al., Benchmarking geant4 nuclear models for hadron therapy with $95 \mathrm{MeV} /$ nucleon carbon ions, Phys. Rev. C, vol. 89, issue 5, May 2014, Art. No. 054616.

[9] C. Divay, et al., Differential cross section measurements for hadron therapy: $50 \mathrm{MeV} /$ nucleon ${ }^{12} \mathrm{C}$ reactions on $\mathrm{H}, \mathrm{C}, \mathrm{O}, \mathrm{Al}$, and ${ }^{\text {nat }} \mathrm{Ti}$ targets, Phys. Rev. C, vol. 95 issue 4, April 2017044602.

[10] T. Toshito, et al., Measurements of total and partial charge changing cross sections for $200-$ to $400-\mathrm{MeV} /$ nucleon $\mathrm{C} 12$ on water and polycarbonate, Phys. Rev. C, vol. 75, May 2007, Art. No. 054606.

[11] C. Zeitlin, et al., Fragmentation cross sections of $290-\mathrm{MeV} /$ nucleon and 400-MeV/nucleon C-12 beams on elemental targets, Phys. Rev. C, vol. 76, issue 1, July 2007, Art. No. 014911.

[12] M. De Napoli, et al., Carbon fragmentation measurements and validation of the GEANT4 nuclear reaction models for hadrontherapy, Phys. Med. Biol., vol. 57, No. 22, pp. 7651--7671, November 2012.

[13] M. Rovituso, et al., Fragmentation of 120 and $200 \mathrm{MeV} / \mathrm{u} 4 \mathrm{He}$ ions in water and PMMA targets, Phys. Med. Biol., vol. 62, No. 4, pp. 13101326, January 2017.

[14] V. Patera, et al., The foot (fragmentation of target) experiment. The 26th International Nuclear Physics Conference, vol. 281, pp. 128, May 2017.

[15] G. Traini, et al., Performance of the ToF detectors in the FOOT experiment, IL NUOVO CIMENTO, 43 C 16, March 2020.

[16] Y. Sun, et al., Design and construction of a time-of-flight wall detector at External Target Facility of HIRFL-CSR, Nuclear Inst. and Methods in Physics Research, A, vol. 893, pp. 68--74, June 2018.

[17] P. S. Marrocchesi, et al., Beam test performance of a scintillator-based detector for the charge identification of relativistic ions, Nuclear Inst. and Methods in Physics Research, A, vol. 659, issue 1, pp. 477--483, December 2011.

[18] A. Rizzo, et al., A compact Time-Of-Flight detector for space applications: The LIDAL system, Nuclear Instruments and Methods in Physics Research A, vol. 898, pp. 98--104, August 2018.

[19] J. B. Birks, Scintillations from Organic Crystals: Specific Fluorescence and Relative Response to Different Radiations, Proc. Phys. Soc. Section A, Vol. 64, No. 10, pp. 874-877, October 1951.

[20] M. Morrocchi, et al., Development and characterization of a $\Delta \mathrm{E}-\mathrm{TOF}$ detector prototype for the FOOT Experiment, Nuclear Inst. and Methods in Physics Research, A, vol. 916, pp. 116-124, February 2019.

[21] E. Ciarrocchi, et al., The $\triangle \mathrm{E}-\mathrm{TOF}$ detector of the FOOT experiment: Experimental tests and Monte Carlo simulations, Nuclear Inst. and Methods in Physics Research, A, vol. 936, pp. 78-79, August 2019.

[22] A. C. Kraan, et al., Charge identification performance of a $\Delta \mathrm{E}-\mathrm{TOF}$ detector prototype for the FOOT experiment, Nuclear Inst. and Methods in Physics Research, A, vol. 958, February 2020, Art. no. 162422.

[23] L. Galli, et al., Fragment charge identification technique with a plastic scintillator detector using clinical carbon beams, Nuclear Inst. and Methods in Physics Research, A, vol. 953, February 2020, Art. no. 163146.

[24] L. Galli, et al., WaveDAQ: An highly integrated trigger and data acquisition system, Nuclear Inst. and Methods in Physics Research, A, vol. 936, pp. 399-400, August 2019.

[25] S. Ritt, et al., Application of the DRS chip for fast waveform digitizing, Nuclear Inst. and Methods in Physics Research, A, vol. 623, issue 1, pp. 486--488, November 2010.

[26] J. Adam, et al., The MEG detector for $\mu+\rightarrow e+\gamma$ decay search, Eur. Phys. J. C, vol. 73, March 2013, Art. No. 2365.

[27] A. Stoykov, et al., On the limited amplitude resolution of multipixel Geiger-mode APDs, Journal of Instrumentation, vol. 2, June 2007, Art. No. P06005.

[28] G. Battistoni, et al., Overview of the FLUKA code, Annals of nuclear energy, vol. 82, pp. 10-18, August 2015.

[29] A. Mirandola, et al., Dosimetric commissioning and quality assurance of scanned ion beams at the Italian National Center for Oncological Hadrontherapy, Med. Phys., Vol. 42 no. 9, pp. 5287-5300, September 2015.
[30] D. Broggio, et al., Polyvinyltoluene scintillators for relative ion dosimetry: An investigation with Helium, Carbon and Neon beams, Nuclear Instruments and Methods in Physics Research B, vol. 254, issue 1, pp. 3--9, January 2007. 